\title{
Beamforming Optimization for Full-Duplex Cooperative Cognitive Radio Networks
}

\author{
Shiyang Hu, Zhiguo Ding, Qiang Ni, Yi Yuan \\ School of Computing and Communications \\ Lancaster University \\ Lancaster, UK \\ \{s.hu, z.ding, q.ni, y.yuan3\}@lancaster.ac.uk
}

\begin{abstract}
This paper considers the problem of beamforming optimization in a cognitive cooperative energy harvesting network, in which the secondary transmitter (ST) harvests energy from the primary transmitter (PT) and relays the information for the primary user (PU) with amplify-and-forward (AF) relay protocol. When the channel of the primary system is affected with deep fading or shadowing effects, the ST can assist the primary information transmission. It is particularly useful to employ the energy harvesting protocol to avoid that the ST does not have enough energy to assist the PU. Based on the self-energy recycling relay protocol, we study the beamforming optimization problem. We develop a semidefinite programming (SDP) relaxation method to solve the proposed problem. We also use SDP and one-dimension (1-D) optimization to solve the beamforming optimization based on a time-switching relaying protocol (TSR) as a benchmark. The simulation results are presented to verify that the self-energy recycling protocol achieves a significant rate gain compared to the TSR protocol and the power-splitting relaying (PSR) protocol.
\end{abstract}

Keywords-beamforming optimization; cooperative cognitive radio networks; energy harvesting

\section{INTRODUCTION}

The cooperation between the primary system and the secondary system in a cognitive radio network was illustrated to improve the spectrum efficiency [1]. When the PU's rate requirement can not be met, the secondary system assists to relay the primary information for the PU and also uses the primary spectrum. In [2], a three phase transmission protocol was proposed. In [3], multiple antennas was employed at the ST, which leads to that the cooperation only requires two phase. However, most works only considered the cooperation on the information level. If the ST is willing to assist the primary transmission but face a practical problem that its own energy can not support the cooperation, the cooperation will not be completed. In order to solve this problem, we introduce the emerging wireless energy harvesting technology into the cooperative cognitive radio network.

The wireless energy harvesting technology is an effective way to prolong the lifetime for the communication system [4]. The meaning of energy harvesting is wirelessly recharging batteries from the external power source. Recently, wireless energy harvesting via the radio-frequency (RF) signal has

978-1-5090-1749-2/16/\$31.00 (C)2016 IEEE drawn a lot of attention [5]. But in the practical implementation of wireless energy harvesting, simultaneously decoding information and harvesting energy is unpractical due to the circuit limitation. Hence the authors in [6] proposed a potential structure for the receiver which harvests energy and detects information separately according a TSR protocol or a PSR protocol. Based on the energy harvesting protocols, there are some works considering energy harvesting problems in the point-to-point communication network [5]. And some works have studied energy harvesting protocols in the wireless cooperative network. In [7], the authors studied the outage probability and the throughput in an amplify-to-forward (AF) cooperative network with an energy harvesting relay node. The authors in [8] proposed a model of the energy harvesting cooperative networks with source-to-destination pairs and one energy harvesting relay node. The difference of some power allocation strategies has also been considered in [8].

Motivated by [9], we study the beamforming optimization problem based on the self-energy recycling relaying protocol in a cooperative cognitive radio network. We modeled an energy harvesting cooperative cognitive radio network with a self-energy recycling node. We formulated the beamforming optimization problem for maximizing the achievable SU's rate subject the available transmitted power at the ST and the minimum PU's rate. We used the SDP relaxation approach to solve it. Simulation results are provided to verify that the self-energy recycling relaying protocol achieves an obvious rate gain compared to TSR or PSR.

Notations: The bold uppercase and lowercase letters are denoted as matrices and vectors, respectively. $\mathcal{E}(\cdot)$ is the expectation over the random variables within the bracket. $(\cdot)^{*},(\cdot)^{T}$ and $(\cdot)^{H}$ are denoted as conjugate, transpose and conjugate and transpose, respectively. $\mathbf{I}_{N}$ denotes $N \times N$ identity matrix. $\operatorname{Tr}(\cdot)$ is the trace of a matrix. vec is the matrix vectorization. $\otimes$ represents the Kronecker operator. $|x|$ denotes the absolution value of a scalar. $\|\mathbf{x}\|^{2}$ is the Frobenius norm. $\mathbb{C}^{M \times N}$ denotes the space of $M \times N$ matrices with complex entries.

\section{SySTEM MODEL}

The cognitive relay network considered in this paper includes a primary system and a secondary system. The secondary system consists of a secondary transmitter-receiver 
pair. The primary system has a primary transmitter-receiver pair, which intends to communicate in a licensed frequency band. We assume that the link between the PT and PU is affected with deep fading or shadowing effects, hence in order to meet the primary system's rate requirement, the ST assists to relay the primary system's information. The ST is an energy harvesting node [7], which has an initial energy and further harvests energy from the PT. The ST receives the PT's information and energy, it uses the harvested energy together with its own energy to transmit the information to the SU and to assist the primary system. The battery capacity of the ST is assumed as infinite. The AF scheme is employed in the ST. A self-energy recycling protocol is considered at the energy harvesting node. The detailed analysis based on this protocol is given in the following section.

Except the ST, all nodes are equipped one antenna. The ST has two groups of the RF chains, one is used for transmitting while the other one is used for receiving. The number of transmission antennas $N_{t}$ and the number of receiving antennas $N_{r}$ are same, i.e. $N_{t}=N_{r}=N$. The perfect channel state information is assumed available at the secondary transmitter. Channels are modeled as quasi-static block fading channels.

\section{BeAmforming With SELF-ENERgy RECyCling PROTOCOL}

\section{A. Self-Energy Recycling Protocol and Transmission Model}

The whole transmission process is operated in the block time, denoted by $T$. Without loss of generality, $T$ is normalized to be unity. The information transmission process is split into two phases. In the first phase, the PT sends information to the ST for $T / 2$ time. In the second phase, the ST transmits the amplified primary system's information and its own information to users with its transmitting antenna. Meanwhile, the ST receives RF signals to harvest energy from the PT with its receiving antenna.

The ST first receives the information from the PT with its receiving antenna. The received information can be expressed as

$$
y_{r}^{1}=\sqrt{P_{p}} \mathbf{h} x_{p}+\mathbf{n}_{\mathbf{r}}^{\mathbf{1}},
$$

where $P_{p}$ is the transmitted power at the PT, $\mathbf{h} \in \mathbb{C}^{N \times 1}$ is the channel vector from the PT to receiving antennas of the $\mathrm{ST}$, and $x_{p}$ is the transmitted information from the PT with $\mathcal{E}\left(\left|x_{p}\right|^{2}\right)=1$ and $\mathbf{n}_{\mathbf{r}}^{\mathbf{1}}$ is the additive complex Gaussian noise vector at the relay node following $\mathcal{C N}\left(\mathbf{0}, \sigma_{r 1}^{2} \mathbf{I}_{N}\right)$.

After the ST receives the information from the PT, it amplifies the received information with the precoding. The ST superimposes the amplified information for the PU and its own information using the cognitive beamforming vector for the SU. The superimposable information can be expressed as

$$
x_{r}=\sqrt{P_{p}} \mathbf{W h} x_{p}+\mathbf{W} \mathbf{n}_{\mathbf{r}}^{\mathbf{1}}+\mathbf{w}_{\mathbf{s}} x_{s},
$$

where $\mathbf{W} \in \mathbb{C}^{\mathbf{N} \times \mathbf{N}}$ is the precoding matrix, $\mathbf{w}_{\mathbf{s}} \in \mathbb{C}^{N \times 1}$ is the cognitive beamforming vector and $x_{s}$ is the information for the primary user with $\mathcal{E}\left(\left|x_{s}\right|^{2}\right)=1$.

The power of the transmitted signal at the ST is

$$
P_{r}=P_{p}\|\mathbf{W h}\|^{2}+\sigma_{r 1}^{2}\|\mathbf{W}\|^{2}+\left\|\mathbf{w}_{\mathbf{s}}\right\|^{2},
$$

In the second phase, the ST uses its transmission antennas to transmit the information to the PU and the SU. The received signal at the PU is given by

$$
y_{p}=\sqrt{P_{p}} \mathbf{g}_{\mathbf{p}}{ }^{H} \mathbf{W h} x_{p}+\mathbf{g}_{\mathbf{p}}{ }^{H} \mathbf{W} \mathbf{n}_{\mathbf{r}}^{\mathbf{1}}+\mathbf{g}_{\mathbf{p}}{ }^{H} \mathbf{w}_{\mathbf{s}} x_{s}+n_{p},
$$

where $\mathbf{g}_{\mathbf{p}} \in \mathbb{C}^{N \times 1}$ is the channel vector between the transmission antennas of the $\mathrm{sST}$ and the PU and $n_{p}$ is additive Gaussian noise at the destination following $\mathcal{C N}\left(0, \sigma_{p}^{2}\right)$.

The received signal at the $\mathrm{SU}$ is given by

$$
y_{s}=\sqrt{P_{p}} \mathbf{g}_{\mathbf{s}}{ }^{H} \mathbf{W h} x_{p}+\mathbf{g}_{\mathbf{s}}{ }^{H} \mathbf{W} \mathbf{n}_{\mathbf{r}}^{\mathbf{1}}+\mathbf{g}_{\mathbf{s}}{ }^{H} \mathbf{w}_{\mathbf{s}} x_{s}+n_{s},
$$

where g $_{\mathrm{s}} \in \mathbb{C}^{N \times 1}$ is the channel vector between the transmission antennas of the ST and the PU and $n_{s}$ is additive Gaussian noise at the destination following $\mathcal{C N}\left(0, \sigma_{s}^{2}\right)$.

Concurrently, the ST is wireless powered by the PT with the dedicated energy-bearing signal. The received signal at the $\mathrm{ST}$ is

$$
\begin{aligned}
y_{r}^{2} & =\sqrt{P_{p}} \mathbf{h} x_{e}+\mathbf{H} \mathbf{x}_{\mathbf{r}}+\mathbf{n}_{\mathbf{r}}^{\mathbf{2}}, \\
& =\sqrt{P_{p}} \mathbf{h} x_{e}+\sqrt{P_{p}} \mathbf{H W h} x_{p}+\mathbf{H W} \mathbf{n}_{\mathbf{r}}^{\mathbf{1}}+\mathbf{H w}_{\mathbf{s}} x_{s}+\mathbf{n}_{\mathbf{r}}^{\mathbf{2}},(6)
\end{aligned}
$$

where $x_{e}$ is the transmitted signal from the PT with $\mathcal{E}\left(\left|x_{e}\right|^{2}\right)=$ $1, \mathbf{H} \in \mathbb{C}^{\mathbf{N} \times \mathbf{N}}$ is the channel matrix of the loop channel at the ST and $\mathbf{n}_{\mathbf{r}}^{2}$ is the received additive complex Gaussian noise vector following $\mathcal{C N}\left(\mathbf{0}, \sigma_{r 2}^{2} \mathbf{I}_{N}\right)$. The amount of the harvested energy at the ST is given by

$$
\begin{aligned}
E=\frac{\eta T}{2} & \left(P_{p}\|\mathbf{h}\|^{2}+P_{p}\|\mathbf{H} \mathbf{W h}\|^{2}+\sigma_{r 1}^{2}\|\mathbf{H W}\|^{2}\right. \\
& \left.+\left\|\mathbf{H w}_{\mathbf{s}}\right\|^{2}+\sigma_{r 2}^{2}\right)
\end{aligned}
$$

where $\eta$ is the energy conversion coefficient at the ST. Then the total available transmit power at the $\mathrm{ST}$ is $\frac{E}{T / 2}$, which can be expressed as

$$
\begin{aligned}
P_{r}^{\max }= & \eta\left(P_{p}\|\mathbf{h}\|^{2}+P_{p}\|\mathbf{H W h}\|^{2}+\sigma_{r 1}^{2}\|\mathbf{H W}\|^{2}\right. \\
& \left.+\left\|\mathbf{H w}_{\mathbf{s}}\right\|^{2}+\sigma_{r 2}^{2}\right)+P_{r}^{i n i},
\end{aligned}
$$

where $P_{r}^{i n i}$ is the initial energy at the ST.

The signal-to-interference plus noise ratio (SINR) at the PU is

$$
S N R_{p}=\frac{P_{p}\left|\mathbf{g}_{\mathbf{p}}{ }^{H} \mathbf{W h}\right|^{2}}{\sigma_{r 1}^{2} \| \mathbf{g}_{\mathbf{p}}{ }^{H} \mathbf{W}||^{2}+\left|\mathbf{g}_{\mathbf{p}}{ }^{H} \mathbf{w}_{\mathbf{s}}\right|^{2}+\sigma_{p}^{2}},
$$

The achievable rate of the PU can be presented as

$$
R_{p}=\frac{1}{2} \log _{2}\left(1+S N R_{p}\right)
$$

The SINR at the SU is

$$
S N R_{s}=\frac{\left|\mathbf{g}_{\mathbf{s}}{ }^{H} \mathbf{w}_{\mathbf{s}}\right|^{2}}{P_{p}\left|\mathbf{g}_{\mathbf{s}}{ }^{H} \mathbf{W h}\right|^{2}+\sigma_{r 1}^{2}\left\|\mathbf{g}_{\mathbf{s}}{ }^{H} \mathbf{W}\right\|^{2}+\sigma_{s}^{2}},
$$

The achievable rate of the SU can be presented as

$$
R_{s}=\frac{1}{2} \log _{2}\left(1+S N R_{s}\right),
$$




\section{B. Problem Formulation and Beamforming Design}

Our objective is to maximize the SU rate subject to the PU's rate and transmit power constraint by jointly optimizing the precoding and the cognitive beamforming. By using the monotonicity, the original problem can be expressed as

$$
\begin{array}{cc}
\max _{\mathbf{W}, \mathbf{w}_{\mathbf{s}}} & \frac{\left|\mathbf{g}_{\mathbf{s}}{ }^{H} \mathbf{w}_{\mathbf{s}}\right|^{2}}{P_{p}\left|\mathbf{g}_{\mathbf{s}}{ }^{H} \mathbf{W h}\right|^{2}+\sigma_{r 1}^{2}\left\|\mathbf{g}_{\mathbf{s}}{ }^{H} \mathbf{W}\right\|^{2}+\sigma_{s}^{2}} \\
\text { s.t. } & \frac{P_{p}\left|\mathbf{g}_{\mathbf{p}}{ }^{H} \mathbf{W h}\right|^{2}}{\sigma_{r 1}^{2}\left\|\mathbf{g}_{\mathbf{p}}{ }^{H} \mathbf{W}\right\|^{2}+\left|\mathbf{g}_{\mathbf{p}}{ }^{H} \mathbf{w}_{\mathbf{s}}\right|^{2}+\sigma_{p}^{2}} \geq \gamma_{p}, \\
& P_{p}\|\mathbf{W h}\|^{2}+\sigma_{r 1}^{2}\|\mathbf{W}\|^{2}+\left\|\mathbf{w}_{\mathbf{s}}\right\|^{2} \\
\leq & \eta\left(P_{p}\|\mathbf{h}\|^{2}+P_{p}\|\mathbf{H W h}\|^{2}+\sigma_{r 1}^{2}\|\mathbf{H W}\|^{2}\right. \\
& \left.+\left\|\mathbf{H} \mathbf{w}_{\mathbf{s}}\right\|^{2}+\sigma_{r 2}^{2}\right)+p_{r}^{i n i},
\end{array}
$$

where $r_{p}$ is the rate requirement for the primary user.

In the above problem, the precoding matrix $\mathbf{W}$ can be characterized as $\mathbf{W}=\mathbf{w}_{\mathbf{p}} \mathbf{h}^{H}$. It can be proved this structure is the optimal structure of $\mathbf{W}$ [10]. This structure amplifies the received signal from the primary transmitter employing $\mathbf{h}^{H}$ to produce a noisy version of the primary information and forward the information with the beamforming $\mathbf{w}_{\mathbf{p}}$. The problem can be further expressed as

$$
\begin{array}{ll}
\max _{\mathbf{w}_{\mathbf{p}}, \mathbf{w}_{\mathbf{s}}} & \frac{\left|\mathbf{g}_{\mathbf{s}}{ }^{H} \mathbf{w}_{\mathbf{s}}\right|^{2}}{\left(P_{p}\|\mathbf{h}\|^{4}+\sigma_{r 1}^{2}\|\mathbf{h}\|^{2}\right)\left|\mathbf{g}_{\mathbf{s}}{ }^{H} \mathbf{w}_{\mathbf{p}}\right|^{2}+\sigma_{s}^{2}} \\
\text { s.t. } & \left(P_{p}\|\mathbf{h}\|^{4}-\gamma_{p} \sigma_{r 1}^{2}\|\mathbf{h}\|^{2}\right)\left|\mathbf{g}_{\mathbf{p}}{ }^{H} \mathbf{w}_{\mathbf{p}}\right|^{2}-\gamma_{p}\left|\mathbf{g}_{\mathbf{p}}{ }^{H} \mathbf{w}_{\mathbf{s}}\right|^{2} \geq \gamma \sigma_{p}^{2}, \\
& \left(P_{p}\|\mathbf{h}\|^{4}+\sigma_{r 1}^{2}\|\mathbf{h}\|^{2}\right)\left(\left\|\mathbf{w}_{\mathbf{p}}\right\|^{2}-\eta\left\|\mathbf{H} \mathbf{w}_{\mathbf{p}}\right\|^{2}\right) \\
& +\left\|\mathbf{w}_{\mathbf{s}}\right\|^{2}-\eta\left\|\mathbf{H} \mathbf{w}_{\mathbf{s}}\right\|^{2} \leq \eta P_{p}\|\mathbf{h}\|^{2}+\eta \sigma_{r 2}^{2}+P_{r}^{i n i}, \text { (14) }
\end{array}
$$

Define a new variable $\mathbf{X}=\mathbf{w}_{\mathbf{p}} \mathbf{w}_{\mathbf{p}}{ }^{H}$ and $\mathbf{Y}=\mathbf{w}_{\mathbf{s}} \mathbf{w}_{\mathbf{s}}{ }^{H}$, the optimization problem can be reformulated as

$$
\begin{aligned}
\max _{\mathbf{X} \succeq \mathbf{0}, \mathbf{Y} \succeq \mathbf{0}} & \frac{\operatorname{Tr}\left(\mathbf{Q}_{\mathbf{1}} \mathbf{Y}\right)}{\operatorname{Tr}\left(\mathbf{Q}_{\mathbf{2}} \mathbf{X}\right)+\sigma_{s}^{2}} \\
\text { s.t. } & \operatorname{Tr}\left(\mathbf{Q}_{\mathbf{3}} \mathbf{X}\right)-\operatorname{Tr}\left(\mathbf{Q}_{\mathbf{4}} \mathbf{Y}\right) \geq \gamma_{p} \sigma_{p}^{2}, \\
& \operatorname{Tr}\left(\mathbf{Q}_{\mathbf{5}} \mathbf{X}\right)+\operatorname{Tr}\left(\mathbf{Q}_{\mathbf{6}} \mathbf{Y}\right) \leq \eta P_{p}\|\mathbf{h}\|^{2}+\eta \sigma_{r 2}^{2}+P_{r}^{i n i}, \\
& \operatorname{Rank}(\mathbf{X})=1 \quad \operatorname{Rank}(\mathbf{Y})=1,
\end{aligned}
$$

where $\mathbf{Q}_{\mathbf{1}}=\mathbf{g}_{\mathbf{s}} \mathbf{g}_{\mathbf{s}}{ }^{H}, \mathbf{Q}_{\mathbf{2}}=\left(P_{p}\|\mathbf{h}\|^{4}+\sigma_{r 1}^{2}\|\mathbf{h}\|^{2}\right) \mathbf{g}_{\mathbf{s}} \mathbf{g}_{\mathbf{s}}{ }^{H}$, $\mathbf{Q}_{\mathbf{3}}=\left(P_{p}\|\mathbf{h} \mid\|^{4}-\gamma_{p} \sigma_{r 1}^{2}\|\mathbf{h}\|^{2}\right) \mathbf{g}_{\mathbf{p}} \mathbf{g}_{\mathbf{p}}{ }^{H}, \mathbf{Q}_{\mathbf{4}}=\gamma_{p} \mathbf{g}_{\mathbf{p}} \mathbf{g}_{\mathbf{p}}{ }^{H}$, $\mathbf{Q}_{\mathbf{5}}=\left(P_{p}\|\mathbf{h}\|^{4}+\sigma_{r 1}^{2}\|\mathbf{h}\|^{2}\right)\left(\mathbf{I}-\eta \mathbf{H}^{H} \mathbf{H}\right)$ and $\mathbf{Q}_{\mathbf{6}}=\mathbf{I}-$ $\eta \mathbf{H}^{H} \mathbf{H}$.

Then we drop the rank-one constraint and then the above problem is a quasi-convex problem. We use the CharnesCooper transformation [11] to solve it. Specifically, we define a new variable $t=\frac{1}{\operatorname{Tr}\left(\mathbf{Q}_{2} \mathbf{X}\right)+\sigma_{s}^{2}}$ and let $\widetilde{\mathbf{X}}=t \mathbf{X}, \widetilde{\mathbf{Y}}=t \mathbf{Y}$. The problem can be recast as follows

$$
\begin{aligned}
\max _{\widetilde{\mathbf{X}} \succeq \mathbf{0}, \widetilde{\mathbf{Y}} \succeq \mathbf{0}, \mathbf{t} \geq \mathbf{0}} \operatorname{Tr}\left(\mathbf{Q}_{\mathbf{1}} \widetilde{\mathbf{Y}}\right) \\
\text { s.t. } \quad \operatorname{Tr}\left(\mathbf{Q}_{\mathbf{2}} \widetilde{\mathbf{X}}\right)+t \sigma_{s}^{2}=1, \\
\\
\operatorname{Tr}\left(\mathbf{Q}_{\mathbf{3}} \widetilde{\mathbf{X}}\right)-\operatorname{Tr}\left(\mathbf{Q}_{\mathbf{4}} \widetilde{\mathbf{Y}}\right) \geq t \gamma_{p} \sigma_{p}^{2}, \\
\operatorname{Tr}\left(\mathbf{Q}_{\mathbf{5}} \widetilde{\mathbf{X}}\right)+\operatorname{Tr}\left(\mathbf{Q}_{\mathbf{6}} \widetilde{\mathbf{Y}}\right) \leq t \eta P_{p}\|\mathbf{h}\|^{2}+t \eta \sigma_{r 2}^{2}+t P_{r}^{i n i},
\end{aligned}
$$

Then the problem is a standard SDP problem and one can efficiently find its optimal solution [12]. The optimal solution is denoted by $\widetilde{\mathbf{X}}^{\star}, \widetilde{\mathbf{Y}}^{\star}, t^{\star}$. Then the solution of $\mathbf{X}$ denoted by $\mathbf{X}^{\star}$ is obtained by $\mathbf{X}^{\star}=\frac{\widetilde{\mathbf{X}}^{\star}}{t^{\star}}$, and the solution of $\mathbf{Y}$ denoted by $\mathbf{Y}^{\star}$ is obtained by $\mathbf{Y}^{\star}=\frac{\widetilde{\mathbf{Y}}^{\star}}{t^{\star}}$. If $\mathbf{X}^{\star}$ and $\mathbf{Y}^{\star}$ are rank one, $\mathbf{w}_{\mathbf{p}}$ and $\mathbf{w}_{\mathbf{s}}$ can be exactly computed via eigenvalue decomposition. Otherwise we can use the Shapiro-BarvinokPataki (SBP) rank reduction theorem [13] to obtain the rankone solution.

\section{BeAmforming With Time-Switching Relaying PROTOCOL}

\section{A. Time Switching Protocol and Transmission Model}

In the time switching protocol, the information transmission process is split into three phases. In the first phase, the PT transmits energy-bearing signals to power the ST for $\alpha T$ and $0 \leq \alpha \leq 1$. During the rest of the block time $(1-\alpha) T$, the primary system's information is transmitted from the PT to the ST. Then the ST forwards the primary system's information and transmits its own information.

The ST harvests the energy in the first phase. The received signal at the ST can be expressed as follows

$$
y_{r}^{1}=\sqrt{P_{p}} \mathbf{h} x_{e}+\mathbf{n}_{\mathbf{r}}^{\mathbf{1}},
$$

where $P_{p}, \mathbf{h}, x_{e}$ and $\mathbf{n}_{\mathbf{r}}^{\mathbf{1}}$ are defined as same as above.

The ST harvests energy from the PT for $\alpha$ time, and the amount of the harvested energy is given by

$$
E=\eta \alpha\left(P_{p}\left\|\mathbf{h}_{\mathbf{r}}\right\|^{2}+\sigma_{r 1}^{2}\right),
$$

where $\eta$ is the energy conversion coefficient. Therefore, the total available transmitted power at the ST is then expressed as follows:

$$
P_{r}^{\max }=\frac{2 \eta \alpha\left(P_{p}\|\mathbf{h}\|^{2}+\sigma_{r 1}^{2}\right)}{1-\alpha},
$$

After the energy transmission, the received information from the PT to the ST can be expressed as

$$
y_{r}^{2}=\sqrt{P_{p}} \mathbf{h} x_{p}+\mathbf{n}_{\mathbf{r}}^{2}+\mathbf{n}_{\mathbf{c}},
$$

where $x_{p}$ and $\mathbf{n}_{\mathbf{r}}^{2}$ are defined as same as above, $\mathbf{n}_{\mathbf{c}}$ is the additive complex Gaussian noise vector due to the RF to baseband conversion following $\mathcal{C N}\left(\mathbf{0}, \sigma_{c}^{2} \mathbf{I}_{N}\right)$.

The signal transmitted by the ST can be written as follows:

$$
x_{r}=\sqrt{P_{p}} \mathbf{W h} x_{p}+\mathbf{W} \mathbf{n}_{\mathbf{r}}^{2}+\mathbf{W} \mathbf{n}_{\mathbf{c}}+\mathbf{w}_{\mathbf{s}} x_{s},
$$

where $\mathbf{W}, \mathbf{w}_{\mathbf{s}}$ and $x_{s}$ are defined as same as above.

The ST's transmission power is given by

$$
P_{r}=P_{p}\|\mathbf{W h}\|^{2}+\sigma_{r 2}^{2}\|\mathbf{W}\|^{2}+\sigma_{c}^{2}\|\mathbf{W}\|^{2}+\left\|\mathbf{w}_{\mathbf{s}}\right\|^{2},
$$

Then the received signal at the PU is given by

$$
\begin{aligned}
y_{p}= & \sqrt{P_{p}} \mathbf{g}_{\mathbf{p}}{ }^{H} \mathbf{W h} x_{p}+\mathbf{g}_{\mathbf{p}}{ }^{H} \mathbf{W} \mathbf{n}_{\mathbf{r}} \\
& +\mathbf{g}_{\mathbf{p}}{ }^{H} \mathbf{W n}_{\mathbf{c}}+\mathbf{g}_{\mathbf{p}}{ }^{H} \mathbf{w}_{\mathbf{s}} x_{s}+n_{p},
\end{aligned}
$$

where $\mathbf{g}_{\mathbf{p}}$ and $n_{p}$ are defined as same as above.

Similarly, the received signal at the SU is given by

$$
\begin{aligned}
y_{s}= & \sqrt{P_{p}} \mathbf{g}_{\mathbf{s}}{ }^{H} \mathbf{W h} x_{p}+\mathbf{g}_{\mathbf{s}}{ }^{H} \mathbf{W} \mathbf{n}_{\mathbf{r}} \\
& +\mathbf{g}_{\mathbf{s}}{ }^{H} \mathbf{W} \mathbf{n}_{\mathbf{c}}+\mathbf{g}_{\mathbf{s}}{ }^{H} \mathbf{w}_{\mathbf{s}} x_{s}+n_{s},
\end{aligned}
$$

where $\mathbf{g}_{\mathbf{s}}$ and $n_{s}$ are definied as same as above. 
The SINR at the PU is

$$
\begin{aligned}
& S N R_{p}= \\
& \frac{P_{p}\left|\mathbf{g}_{\mathbf{p}}{ }^{H} \mathbf{W h}\right|^{2}}{\sigma_{r 2}^{2}\left\|\mathbf{g}_{\mathbf{p}}{ }^{H} \mathbf{W}\right\|^{2}+\sigma_{c}^{2}\left|\mathbf{g}_{\mathbf{p}}{ }^{H} \mathbf{W}\right|^{2}+\left|\mathbf{g}_{\mathbf{p}}{ }^{H} \mathbf{w}_{\mathbf{s}}\right|^{2}+\sigma_{p}^{2}},
\end{aligned}
$$

The achievable rate of the PU can be presented as

$$
R_{p}=\frac{1-\alpha}{2} \log _{2}\left(1+S N R_{p}\right),
$$

The SINR at the SU is

$$
\begin{aligned}
& S N R_{s}= \\
& \frac{\left|\mathbf{g}_{\mathbf{s}}{ }^{H} \mathbf{w}_{\mathbf{s}}\right|^{2}}{P_{p}\left|\mathbf{g}_{\mathbf{s}}{ }^{H} \mathbf{W h}\right|^{2}+\sigma_{r 2}^{2}\left\|\mathbf{g}_{\mathbf{s}}{ }^{H} \mathbf{W}\right\|^{2}+\left.\sigma_{c}^{2}\left\|\mathbf{g}_{\mathbf{s}}{ }^{H} \mathbf{W}\right\|\right|^{2}+\sigma_{s}^{2}},
\end{aligned}
$$

The achievable rate of the SU can be presented as

$$
R_{s}=\frac{1-\alpha}{2} \log _{2}\left(1+S N R_{s}\right)
$$

\section{B. Problem Formulation and Beamforming Design}

We formulate the optimization problem like the problem in the above section. By using the monotonicity and the optimal precoding matrix $\mathbf{W}=\mathbf{w}_{\mathbf{p}} \mathbf{h}^{H}$ [10], the problem can be expressed by

$$
\begin{array}{ll}
\max _{\mathbf{w}_{\mathbf{p}}, \mathbf{w}_{\mathbf{s}}, \alpha} \frac{\left|\mathbf{g}_{\mathbf{s}}{ }^{H} \mathbf{w}_{\mathbf{s}}\right|^{2}}{\left(P_{p}\|\mathbf{h}\|^{4}+\sigma_{r 2}^{2}\|\mathbf{h}\|^{2}+\left.\sigma_{c}^{2}\|\mathbf{h}\|\right|^{2}\right)\left|\mathbf{g}_{\mathbf{s}}{ }^{H} \mathbf{w}_{\mathbf{p}}\right|^{2}+\sigma_{s}^{2}} \\
\text { s.t. } \quad\left(P_{p}\|\mathbf{h}\|^{4}-\gamma_{p} \sigma_{r 1}^{2}\|\mathbf{h}\|^{2}-\gamma_{p} \sigma_{c}^{2}\|\mathbf{h}\|^{2}\right)\left|\mathbf{g}_{\mathbf{p}}{ }^{H} \mathbf{w}_{\mathbf{p}}\right|^{2} \\
\quad-\gamma_{p}\left|\mathbf{g}_{\mathbf{p}}{ }^{H} \mathbf{w}_{\mathbf{s}}\right|^{2} \geq \gamma \sigma_{p}^{2}, \\
\quad\left(P_{p}\|\mathbf{h}\|^{4}+\sigma_{r 2}^{2}\|\mathbf{h}\|^{2}+\sigma_{c}^{2}\|\mathbf{h}\|^{2}\right)\left\|\mathbf{w}_{\mathbf{p}}\right\|^{2}+\left\|\mathbf{w}_{\mathbf{s}}\right\|^{2} \\
\leq \frac{2 \eta \alpha\left(P_{p}\|\mathbf{h}\|^{2}+\sigma_{r 1}^{2}\right)}{1-\alpha}+p_{r}^{i n i}, \\
0 \leq \alpha \leq 1,
\end{array}
$$

Define a new variable $\mathbf{X}=\mathbf{w}_{\mathbf{p}} \mathbf{w}_{\mathbf{p}}{ }^{H}$ and $\mathbf{Y}=\mathbf{w}_{\mathbf{s}} \mathbf{w}_{\mathbf{s}}{ }^{H}$, the optimization problem can be reformulated as

$$
\begin{aligned}
& \max _{\mathbf{X} \succeq \mathbf{0}, \mathbf{Y} \succeq \mathbf{0}, \alpha} \frac{\operatorname{Tr}\left(\mathbf{Q}_{\mathbf{1}} \mathbf{Y}\right)}{\operatorname{Tr}\left(\mathbf{Q}_{\mathbf{2}} \mathbf{X}\right)+\sigma_{s}^{2}} \\
& \text { s.t. } \operatorname{Tr}\left(\mathbf{Q}_{\mathbf{3}} \mathbf{X}\right)-\operatorname{Tr}\left(\mathbf{Q}_{\mathbf{4}} \mathbf{Y}\right) \geq \gamma_{p} \sigma_{p}^{2}, \\
& \operatorname{Tr}\left(\mathbf{Q}_{\mathbf{5}} \mathbf{X}\right)+\operatorname{Tr}(\mathbf{Y}) \leq \frac{2 \eta \alpha\left(P_{p}\|\mathbf{h}\|^{2}+\sigma_{r 1}^{2}\right)}{1-\alpha}+p_{r}^{i n i}, \\
& 0 \leq \alpha \leq 1, \\
& \operatorname{Rank}(\mathbf{X})=1 \quad \operatorname{Rank}(\mathbf{Y})=1,
\end{aligned}
$$

where $\mathbf{Q}_{\mathbf{1}}=\mathbf{g}_{\mathbf{s}} \mathbf{g}_{\mathbf{s}}{ }^{H}, \quad \mathbf{Q}_{\mathbf{2}}=\left(P_{p}\|\mathbf{h}\|^{4}+\right.$ $\left.\sigma_{r 2}^{2}\|\mathbf{h}\|^{2}+\sigma_{c}^{2}\|\mathbf{h}\|^{2}\right) \mathbf{g}_{\mathbf{s}} \mathbf{g}_{\mathbf{s}}{ }^{H}, \quad \mathbf{Q}_{\mathbf{3}}=\left(P_{p}\|\mathbf{h}\|^{4}-\right.$ $\left.\gamma_{p} \sigma_{r 2}^{2}\|\mathbf{h}\|^{2}-\gamma_{p} \sigma_{c}^{2}\|\mathbf{h}\|^{2}\right) \mathbf{g}_{\mathbf{p}} \mathbf{g}_{\mathbf{p}}{ }^{H}, \quad \mathbf{Q}_{\mathbf{4}}=\gamma_{p} \mathbf{g}_{\mathbf{p}} \mathbf{g}_{\mathbf{p}}{ }^{H}$, $\mathbf{Q}_{\mathbf{5}}=P_{p}\|\mathbf{h}\|^{4}+\sigma_{r 2}^{2}\|\mathbf{h}\|^{2}+\sigma_{c}^{2}\|\mathbf{h}\|^{2}$.

It is worthy to point out that it is still challenging to solve this problem directly, mainly due to the rank one constraint and the time switching coefficient in the problem. In order to tackle these difficulties, we can perform 1-D optimization with respect to the time-switching coefficient. Specifically, for a given time-switching coefficient, the optimal relay beamforming matrix can be efficiently obtained. We perform a full search with respect to the time-switching coefficient, then solve the problem with all possible value of the time-switching coefficient. Once the value of $\alpha$ is fixed, the problem can be treated as a quasi-convex SDP problem, where we drop the rank one constraint. Then we use the Charnes-Cooper transformation [11] to solve it. Specifically, we define a new variable $t=\frac{1}{\operatorname{Tr}\left(\mathbf{Q}_{\mathbf{2}} \mathbf{X}\right)+\sigma_{d}^{2}}$ and let $\widetilde{\mathbf{X}}=t \mathbf{X}, \widetilde{\mathbf{Y}}=t \mathbf{Y}$. The problem can be recast as follows

$$
\begin{array}{ll}
\max _{\widetilde{\mathbf{X}} \succeq \mathbf{0}, \widetilde{\mathbf{Y}} \succeq \mathbf{0}, \mathbf{t} \geq \mathbf{0}} \operatorname{Tr}\left(\mathbf{Q}_{\mathbf{1}} \widetilde{\mathbf{Y}}\right) \\
\text { s.t. } & \operatorname{Tr}\left(\mathbf{Q}_{\mathbf{2}} \widetilde{\mathbf{X}}\right)+t \sigma_{s}^{2}=1, \\
& \operatorname{Tr}\left(\mathbf{Q}_{\mathbf{3}} \widetilde{\mathbf{X}}\right)-\operatorname{Tr}\left(\mathbf{Q}_{\mathbf{4}} \widetilde{\mathbf{Y}}\right) \geq t \gamma_{p} \sigma_{p}^{2}, \\
& \operatorname{Tr}\left(\mathbf{Q}_{\mathbf{5}} \widetilde{\mathbf{X}}\right)+\operatorname{Tr}(\widetilde{\mathbf{Y}}) \leq \frac{2 t \eta \alpha\left(P_{p}\|\mathbf{h}\|^{2}+\sigma_{r 1}^{2}\right)}{1-\alpha}+p_{r}^{i n i},
\end{array}
$$

Then the problem is solvable like the problem with the selfenergy recycling relaying protocol. The optimal solution is denoted by $\widetilde{\mathbf{X}}^{\star}, \widetilde{\mathbf{Y}}^{\star}, t^{\star}$. Then the solution of $\mathbf{X}$ denoted by $\mathbf{X}^{\star}$ is obtained by $\mathbf{X}^{\star}=\frac{\widetilde{\mathbf{X}}^{\star}}{t^{\star}}$, and the solution of $\mathbf{Y}$ denoted by $\mathbf{Y}^{\star}$ is obtained by $\mathbf{Y}^{\star}=\frac{\widetilde{\mathbf{Y}}^{\star}}{t^{\star}}$. If $\mathbf{X}^{\star}$ and $\mathbf{Y}^{\star}$ are not rank one solution, we can also use the SBP rank reduction theorem to obtain the rank-one solution.

It is necessary to mention that we can also use PSR protocol as another benchmark scheme compared with the self-energy recycling scheme. The PSR protocol can be referred as [7]. Our method to optimize the beamforming with the TSR protocol is easily extended to the same optimization problem based on the PSR. Hence we omit its detailed procedure here, and we provide its simulation result in the next section.

\section{NumericAl RESUlts}

In this section, simulation results are provided to evaluate the performance of the proposed beamforming optimization 29 ) model [14]. The loop channel path loss is $-15 \mathrm{~dB}$ [15]. The initial power at the $\mathrm{ST}$ is $10 \mathrm{dBm}$. The minimum requirement rate of the PU is $3 \mathrm{bps} / \mathrm{Hz}$. The distance between each node is set as $10 \mathrm{~m}$. The system bandwidth is $200 \mathrm{kHz}$. The carrier frequency is $470 \mathrm{MHz}$ which is accorded with the IEEE 802.11af Wi-Fi parameters [16]. The noise variance is assumed as $\sigma_{r 1}^{2}=\sigma_{r 2}^{2}=\sigma_{c}^{2}=\sigma_{p}^{2}=\sigma_{s}^{2}=-25 \mathrm{dBm}$. The energy conversion efficient is 0.8 . Simulation results were averaged over 1000 independent trials.

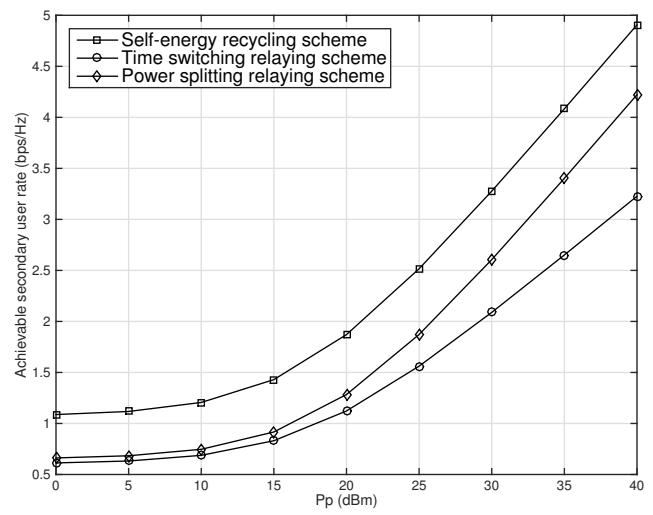

Fig. 1. Achievable SU's rate versus transmitted power at the PT for different energy harvesting protocols. 


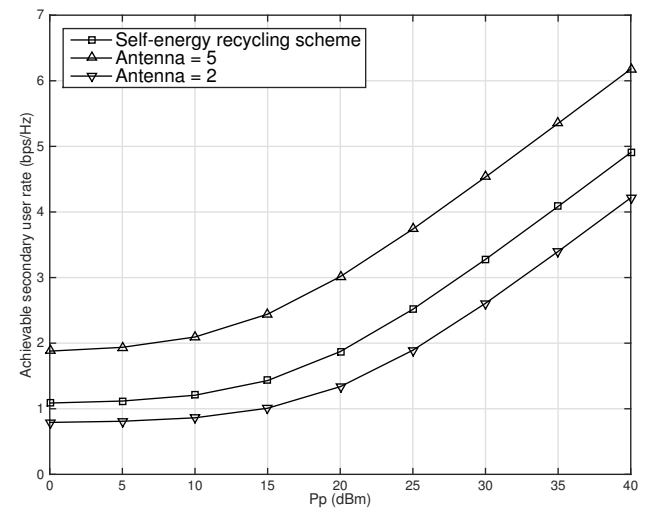

(a) Performance comparison of the self-energy recycling protocol with different numbers of antenna.

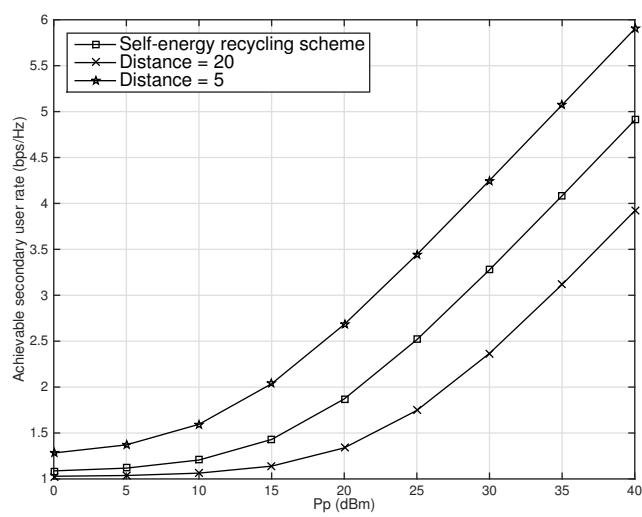

(b) Performance comparison of the self-energy recycling protocol with different distances.

Fig. 2. Achievable SU's rate versus transmitted power at the PT for the self-energy recycling relaying protocol with different parameters.

In Fig. 1, the achievable SU's rates achieved by different energy harvesting schemes versus the transmitted power at the PT are plotted. The result for the self-energy recycling protocol is demonstrated and the results for the TSR scheme and the PSR scheme are also shown as benchmarks. The results for PSR and TSR schemes are obtained with their optimal energy harvesting coefficient. It can be seen that curves of achievable SU's rate for these energy harvesting schemes are monotonically non-decreasing functions of the transmitted power at the PT because the higher transmitted power results in more available power at the ST for the broadcasting transmission. The self-energy recycling scheme outperforms the TSR scheme and PSR scheme. This is because that the full-duplex structure used in the self-energy recycling relaying protocol not only harvests the power transmitted form the PT, but also recycles the part of transmitted power at the ST. Compared to TSR scheme and PSR scheme, the selfenergy harvesting scheme achieves an obvious rate gain. The self-energy recycling protocol has a better performance than the other energy harvesting protocols.

In Fig. 2, we present the achievable SU's rate versus the transmitted power at the PT for the self-energy recycling relaying protocol with different system parameters. It can be seen from the figure that the achievable SU's rate improves with the increased number of the antenna and is degraded with the increase of the distance between each node. This is attributed to the fact that the ST could exploit the array gain to achieve better performance with more antennas and the longer distance makes channel attenuation larger in turns resulting in the worse performance.

\section{CONCLUSIONS}

In this paper, we formulated the beamforming optimization problem in a cooperative cognitive radio network based on the self-energy recycling relaying protocol. We proposed a SDPbased solution to obtain the optimal solution for the proposed problem. In order to demonstrate the advantage of the selfenergy recycling relaying protocol, we also used the SDP relaxation and 1-D optimization to solve the beamforming optimization problem with the TSR protocol and the PSR protocol. Simulation results illustrated that the self-energy recycling relaying protocol could improve the achievable rate compared to TSR scheme and PSR scheme. The trade-off between the achievable rate and system parameters is also provided.

\section{REFERENCES}

[1] S. H. Song and K. B. Letaief, "Prior zero-forcing for relaying primary signals in cognitive network," Proc. IEEE Global Commun. Conf., pp. 1-5, Dec. 2011.

[2] O. Simeone, I. Stanojev, S. Savazzi, Y. Bar-Ness, U. Spagnolini, and R. Pickholtz, "Spectrum leasing to cooperating secondary ad hoc networks," IEEE J. Sel. Areas Commum., vol. 26, no. 1, pp. 203-213, Jan. 2008.

[3] K. Hamdi, K. Zarifi, K. B. Letaief, and A. Ghrayeb, "Beamforming in relay-assisted cognitive redio systems: a convex optimization approach," Proc. IEEE Int. Conf. Commun., pp. 1-5, June 2011.

[4] R. Zhang and C. K. Ho, "MIMO broadcasting for simultaneous wireless information and power transfer," IEEE Trans. Wireless Commun. vol. 12, no. 5, pp. 1989-2001, May 2013.

[5] L. R. Varshney, "Transporting information and energy simultaneously," in Proc. IEEE Int. Symp. Inf. Theory (ISIT), Toronto, Canada, Jul. 2008.

[6] X. Zhou, R. Zhang, and C. K. Ho, "Wireless information and power transfer: Architecture design and rate-energy tradeoff," IEEE Transactions on Communications, (submitted) Available: http://arxiv.org/abs/1205.0618.

[7] A. A. Nasir, X. Zhou, S. Durrani, and R. A. Kennedy, "Relaying protocols for wireless energy harvesting and information processing," IEEE Trans. Wireless Commun., vol. 12, pp. 3622-3636, 2013.

[8] Z. Ding, S. M. Perlaza, I. Esnaola, and H. V. Poor, "Power allocation strategies in energy harvesting wireless cooperative networks," IEEE Trans. Wireless Commun., submitted (available at http://arxiv.org/abs/1307.1630).

[9] Y. Zeng and R. Zhang, "Full-duplex wireless-powered relay with selfenergy recycling," IEEE Wireless Commun. Letters, 2015.

[10] G. Zheng, S. H. Song, K. K. Wong, and B. Ottersten, "Cooperative cognitive networks: optimal, distributed and low-complexity algorithms," IEEE Trans. Signal Process., vol. 61, no. 11, pp. 2778-2790, June 2013.

[11] A. Charnes and W. W. Cooper, "Prgramming with linear fractional functions," Naval Res. Logist. Quart., vol. 9, pp. 181-186, 1962.

[12] M. Grant and S. Boyd, "Cvx: Matlab software for disciplined convex programming," Jun. 2012. Available at http://cvxr.com.cvx.

[13] Y. Huang and D. P. Palomar, "Rank-constrained separable semidefinite programming with applications to optimal beamforming," IEEE Trans. Signal Process., vol. 58, no. 2, pp. 664-678, 2010.

[14] "Tgn channel models," IEEE 802.1103/940r4, Tech. Rep., May 2004.

[15] H. G. Schantz, "Near field propagation law and a novel fundamental limit to antenna gain versus size," IEEE Antennas and Propag. Society Int. Symposium, 2005.

[16] H. S. Chen and W. Gao, "Mac and phy proposal for 802.11af," Tech. Rep., Feb. Available at http://mentor.ieee.org/802.11/dcn/10/11-10-025800-00af-mac-and-phy-proposal-for-802-11af.pdf. 